\title{
Proposal of Heterogeneous Wireless Communication Network with Soft Handover in Application Layer: Feasibility Study Based on Field Trial Results
}

\author{
S. Kameda ${ }^{+}$, H. Oguma ${ }^{+\$}$, N. Izuka ${ }^{\#}$, F. Yamagata ${ }^{+}$, Y. Asano ${ }^{\#}$, Y. Yamazaki ${ }^{\#}$, \\ S. Tanifuji ${ }^{+}$, N. Suematsu ${ }^{+}$, T. Takagi ${ }^{+}$, and K. Tsubouchi ${ }^{+}$ \\ ${ }^{+}$Research Institute of Electrical Communication, Tohoku University \\ ${ }^{\$}$ Miyagi Prefectural Government \\ "Network Service Development Department, Softbank Telecom
}

\begin{abstract}
This paper proposes a heterogeneous wireless communication network with soft handover in the application layer. The proposed network requires no upgrades of wireless infrastructure and mobile terminals to evolve the present homogeneous networks to the proposed heterogeneous network. Only installing application programs on the content server side and the mobile terminal side is required. The performance of the proposed network has been evaluated in a field trial using a mobile broadband wireless access (MBWA) air interface with wide coverage and a wireless local area network (WLAN) air interface with high throughput. The field trial results show that the maximum value of the handover outage time is only $170 \mathrm{~ms}$. The proposed heterogeneous wireless communication network is promising since both high throughput and wide coverage area are attained by a combination of the proposed handover scheme with the present homogeneous wireless networks.
\end{abstract}

Key-words: Heterogeneous Wireless Communication Networks, WLAN, MBWA, Handover

\section{INTRODUCTION}

Signal bandwidth in wireless data communications is being widened with an increase of needs for broadband wireless communication services. However, the main benefit of broadband signal, i.e., high throughput, is received by only users close to the base-station since the transmitter output power is limited. With an increase of the signal bandwidth, the maximum throughput is increased; however, the coverage area of the signal with the maximum throughput is narrowed. To solve the problem, we propose a heterogeneous wireless communication network where both high throughput and wide coverage are attained by using multiple air interfaces.

Many handover schemes [1]-[7] such as vertical handover or media independent handover have been proposed and discussed to carry out handovers across wireless networks with different air interfaces. In the already proposed handover schemes, a soft vertical handover scheme based on mobile internet protocol version 6 (Mobile IPv6), using cross-layer design, can significantly reduce outage time [7]. However, the scheme requires upgrades of wireless infrastructures and mobile terminals. All the handover schemes except the session initiation protocol (SIP) based handover [2] require the upgrades of the infrastructures and terminals since new functions for handover are required to be implemented. The SIP-based scheme requires no infrastructure upgrades; however, the scheme yields a long outage time such as several seconds or several tens of seconds since the scheme is based on hard handover [3]. A soft vertical handover scheme based on SIP has been proposed; however, the scheme requires additional equipment for soft handover [6].

In this paper, we propose a novel soft vertical handover scheme without requiring any upgrades of the infrastructures and terminals. A key to the proposed network is an applicationlayer soft handover scheme. All the proposed handover functions are implemented in the application layer. Thus, no infrastructure upgrades but only application software installations on a server and mobile terminals are required. No additional equipment for soft handover is required in the proposed scheme.

We have carried out a field trial to confirm the feasibility of the proposed heterogeneous network with the application-layer soft handover. The air interfaces are Fast Low-latency Access with Seamless Handoff Orthogonal Frequency Division Multiplexing (FLASH OFDM) [8]-[13] and the Institute of Electrical and Electronics Engineers (IEEE) 802.11g wireless local area network (WLAN). The field trial results show that the proposed network is feasible since the handover outage time is only less than $170 \mathrm{~ms}$, which is tolerable value in a commercial service.

This paper is organized as follows. Section 2 describes the proposed heterogeneous wireless communication network. Section 3 describes the proposed application-layer handover scheme. Section 4 discusses the field trial results of the proposed network. Finally, Section 5 gives the conclusions.

\section{Proposed HETEROGENEOUS WIRELESS COMMUNICATION NETWORK}

This section describes an overview of the proposed heterogeneous wireless communication network.

First, a coverage problem in the present homogeneous mobile broadband wireless access (MBWA) network is discussed. To clarify the problem, the coverage estimates of uplink 64 quadrature amplitude modulation (QAM) signal based on the field trial results are shown under different bandwidth conditions. The same method as 16 QAM coverage estimation [13], which is based on the received signal level, is used to estimate 64 QAM coverage. Figure 1 shows the 


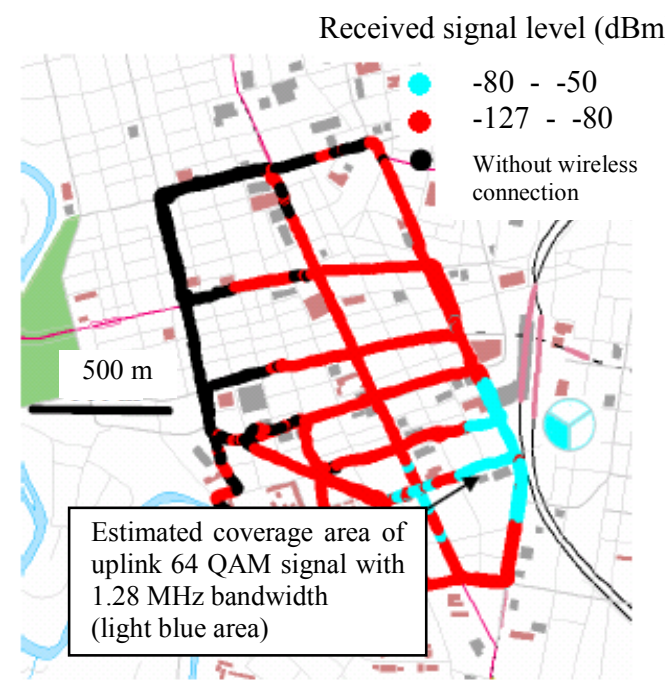

Figure 1 Estimated coverage area of uplink 64 QAM signal with $1.28 \mathrm{MHz}$ bandwidth.

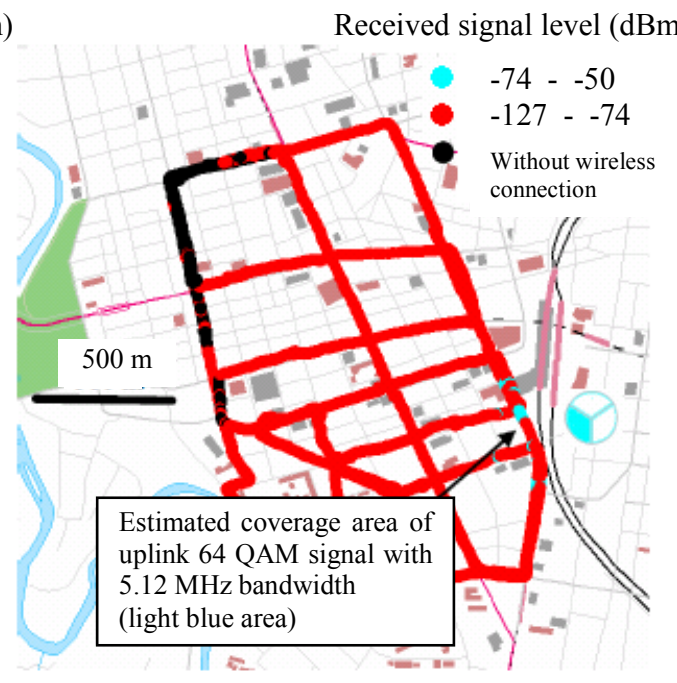

Figure 2 Estimated coverage area of uplink 64 QAM signal with $5.12 \mathrm{MHz}$ bandwidth.

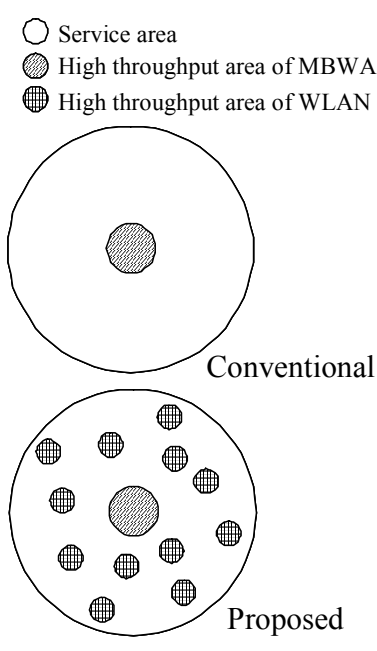

Figure 3 Simplified service areas of conventional homogeneous network and proposed heterogeneous network. estimated coverage area of the uplink 64 QAM signal with the output power of $23 \mathrm{dBm}$ when the signal bandwidth is 1.28 $\mathrm{MHz}$. This figure shows that the estimated coverage is around $300 \mathrm{~m}$. Figure 2 shows the estimated coverage area when the signal bandwidth is $5.12 \mathrm{MHz}$ under the same terminal output power condition as in Fig. 1. These figures show that the quad bandwidth condition yields narrower coverage area. The 64 QAM coverage area is almost disappeared when the signal bandwidth is $5.12 \mathrm{MHz}$. There are no coverage areas of 64 QAM when the signal bandwidths are $10.24 \mathrm{MHz}$ and 20.48 $\mathrm{MHz}$. The above results show that high level modulation, which yields high throughput, can be used in only the area close to the base-station. The results are generalized as follows. Maximum throughput is increased with an increase of signal bandwidth; however, the coverage area of the maximum throughput signal is narrowed. We consider that all homogeneous wireless data communication networks with traditional cellular configuration have this problem since signal bandwidth, coverage, and throughput are common basic parameters in the networks.

Next, the proposed heterogeneous wireless communication network using multiple air interfaces is discussed to solve the coverage problem. Figure 3 shows simplified service areas of the conventional homogeneous MBWA network and the proposed heterogeneous network with MBWA and WLAN air interfaces. High throughput area is limited to the cell center in the homogeneous network. In contrast, high throughput areas except the cell center are provided by the WLAN air interface in the proposed network. This means the proposed heterogeneous network attains a wider service area with high throughput than that obtained in the conventional homogeneous network. The other service areas are provided by the MBWA air interface with relatively low throughput. To provide a wireless data communication service with higher throughput and wide coverage area to users in the proposed network, seamless handover is required between wireless networks with different air interfaces. An application-layer soft handover scheme, which is a key to the proposed network, is described in the next section.

\section{APPLICATION-LAYER SOFT HANDOVER SCHEME}

This section describes the proposed handover scheme in the heterogeneous wireless network.

\section{A. Overview of proposed handover scheme}

In the proposed scheme, the mobile terminal has wide coverage air interface such as MBWA and high throughput air interface such as WLAN. Each air interface has different physical layer, data link layer, and IP layer. All handover functions are implemented in the application layer.

Figure 4 illustrates the proposed handover scheme. The mobile terminal has multiple wireless communication cards. Each communication card independently establishes wireless links with the base-station and the access point. The mobile terminal selects an appropriate air interface from the two interfaces. In the field trial, the simple selection rule that the mobile terminal preferentially selects WLAN was adopted. The content server selects the transmission route which the mobile terminal demands, and transmits data packets along the route.

\section{B. Handover procedure}

The proposed handover procedure is as follows. As an example, the handover procedure is discussed when the mobile terminal moving in the service area of the wide coverage air interface such as MBWA, moves across the service area of the high throughput air interface such as WLAN. Figure 5 shows the mobile terminal out of the WLAN service area. The mobile terminal establishes a communication link with the content server using the MBWA air interface. When the communication link is established, the content server assigns the unique identifier and identification number to the mobile terminal. The server identifies the terminal using them. After identification is completed, the server communicates with the terminal using the MBWA air interface. Note that the identification number is not IP address. When the IP address is used as an identification number in the presence of network 


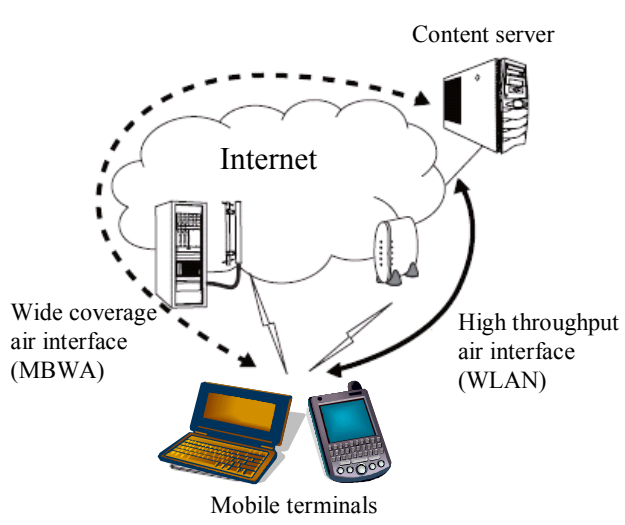

Figure 4 Proposed handover scheme.

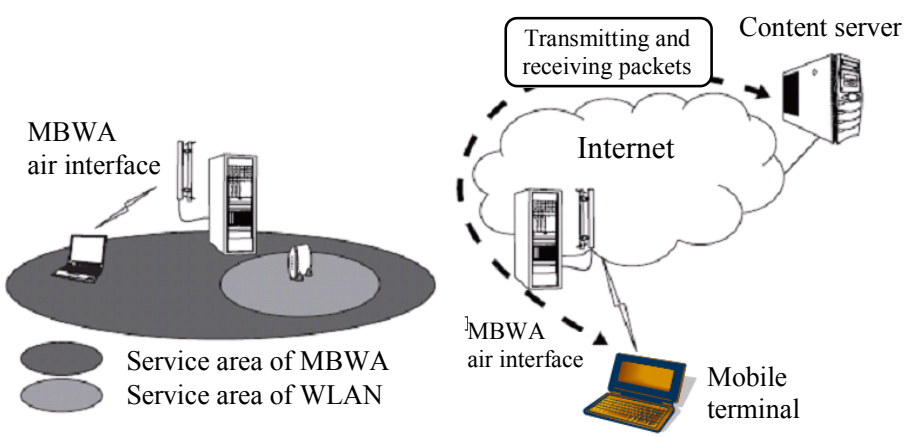

Figure 5 Mobile terminal out of WLAN service area.

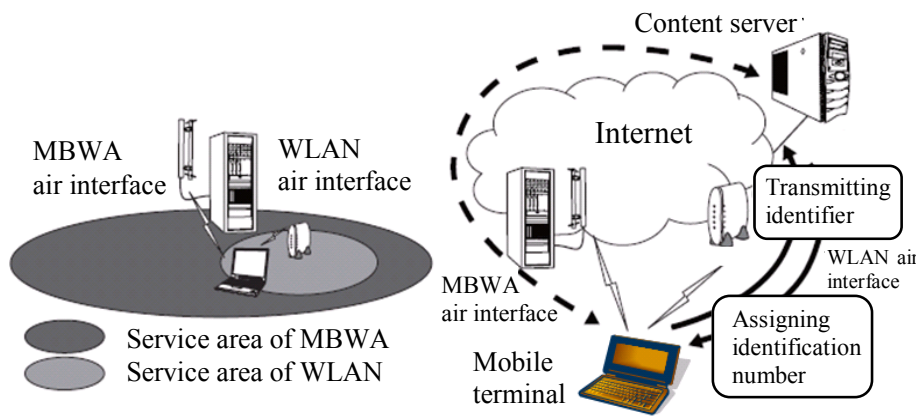

Figure 6 Mobile terminal that has just moved into WLAN service area.

address translation, the proposed handover scheme cannot be carried out.

Figure 6 shows the mobile terminal that has just moved into the WLAN service area. The mobile terminal establishes a communication link with the content server using the WLAN air interface. By receiving the identifier of the terminal through the WLAN air interface, the content server understands that the mobile terminal using the MBWA air interface is the same as that using the WLAN air interface. If required, the server assigns an identifier and an identification number after confirming the presence of one of them.

Figure 7 shows the mobile terminal after identification. Since the content server understands the terminal using the MBWA air interface is the same as that using the WLAN air interface, the terminal can use both the communication links. In the field trial, the terminal selects the WLAN air interface
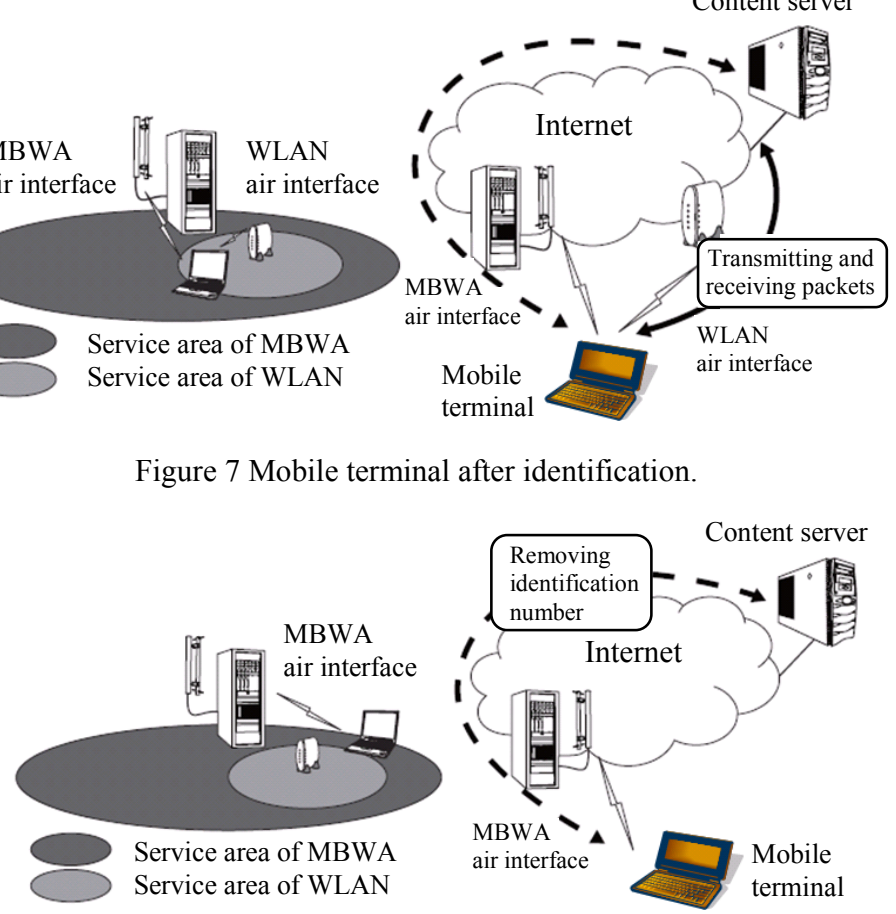

Figure 7 Mobile terminal after identification.

Figure 8 Mobile terminal that has just moved out of WLAN service area.

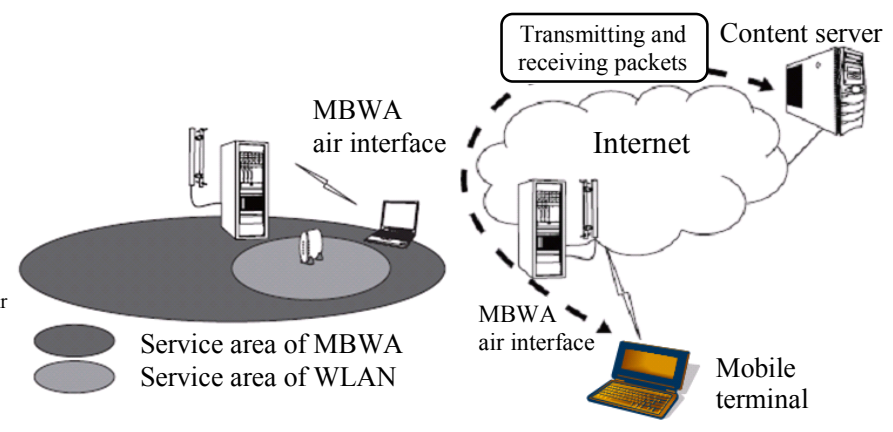

Figure 9 Mobile terminal after moving across WLAN service area.

corresponding to the rule that the mobile terminal preferentially selects the WLAN air interface.

Figure 8 shows the mobile terminal that has just moved out of the WLAN service area. The terminal demands the removal of the WLAN identification number via MBWA after disconnecting the WLAN link. The content server removes the WLAN identification number when the server receives the removal demand from the terminal or after time-out.

Figure 9 shows the mobile terminal after moving across the WLAN service area. The mobile terminal communicates with the content server using the MBWA air interface as the same as shown in Fig. 5.

\section{Implementation of proposed handover scheme}

To carry out the field trial, we have developed application programs of the proposed handover scheme on the content 


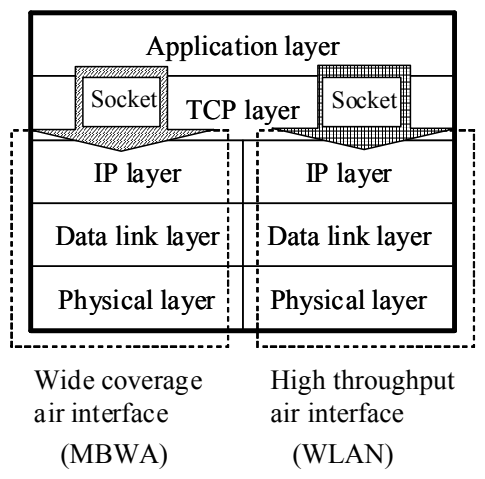

Figure 10 Protocol stack of multiple sockets.

server and mobile terminals. The developed programs use multiple sockets binding each air interface. Figure 10 shows the protocol stack of the multiple sockets. The mobile terminal selects an air interface through selecting a socket to send and receive packets.

\section{Benefits of proposed handover scheme}

The above handover scheme using multiple sockets gives the following benefits to the proposed heterogeneous wireless network.

\section{1) Location management at content server}

In the proposed network, only the content server manages terminal location information which service area the mobile terminal is in. The proposed network does NOT require the present wireless infrastructure such as MBWA and WLAN to manage the location information as a new function for handover. Thus, the present wireless infrastructure can be applied as it is to the proposed network.

\section{2) Any air interface applicable to proposed network}

Since the proposed handover scheme does not require the present wireless infrastructure to add functions for handover, any wireless interfaces can be applied to the proposed network. In the field trial, IEEE 802.11g and FLASH OFDM air interfaces were used; however, any air interfaces such as IEEE $802.11 \mathrm{a} / \mathrm{b} / \mathrm{g} / \mathrm{ac} / \mathrm{ad}$, mobile Worldwide Interoperability for Microwave Access (WiMAX), 3G, Long Time Evolution (LTE), and LTE-advanced can be also used to construct the proposed network. If LTE and 802.11ac air interfaces are used, the proposed network can provide a wireless communication service with high throughput such as $1 \mathrm{Gbit} / \mathrm{s}$ and wide coverage area such as a cell radius of 1 or $2 \mathrm{~km}$ to users though the highest throughput is obtained under a low mobility condition.

3) No upgrades of wireless infrastructures and terminals

Since the handover functions of the proposed scheme are implemented as application programs on the content server and mobile terminals, only installing the programs is required to construct the proposed network. Thus, no upgrades of the present wireless infrastructures and mobile terminals are required to evolve homogeneous networks to the proposed heterogeneous wireless network. We consider that the proposed network is suitable for smart phones with two air interfaces because of the following reasons. (1) Application software can
Table 2 Handover outage times from WLAN to MBWA.

\begin{tabular}{|c|c|}
\hline Measurements & Outage times \\
\hline 1 & $57 \mathrm{~ms}$ \\
\hline 2 & $44 \mathrm{~ms}$ \\
\hline 3 & $112 \mathrm{~ms}$ \\
\hline 4 & $57 \mathrm{~ms}$ \\
\hline average & $67.5 \mathrm{~ms}$ \\
\hline
\end{tabular}

be installed. (2) Additional implementation of air interface is not required.

4) Air interface selection while keeping packet transmission routes alive

In the proposed handover scheme, one of the air interfaces can be selected while keeping all the packet transmission routes alive, using multiple sockets. Air interface selection while keeping the routes alive yields a shorter outage time than that obtained in the conventional SIP-based application layer handover scheme [2], as well as the soft handover schemes based on Mobile IPv6 [7] and SIP [6]. Note that the former requires updates of the infrastructure and terminals, and the latter requires additional equipment for soft handover. In contrast, the proposed handover scheme requires only installations of handover application programs.

\section{Field TRial Results of Proposed Network WITH APPLICATION-LAYER HANDOVER}

This section describes the field trial results of the proposed heterogeneous wireless communication network with the application-layer handover.

\section{A. Measurement conditions}

The measurements were carried out in a building in the field trial area. The building is in both the service areas of MBWA with wide coverage and WLAN with high throughput. The MBWA service is experimentally provided in the field trial area of Sendai city in Japan [9]-[13]. We deployed a WLAN access point in the building. The FLASH OFDM MBWA network was used as a wide coverage air interface, and the IEEE 802.11g WLAN network was used as a high throughput air interface. The operating systems of the mobile terminal and the content server are Linux 2.6.9 and 2.6.14, respectively. The application programs of the proposed scheme on the server and terminal sides were written on Linux.

\section{B. Handover outage times}

Table 1 shows the measured outage times in the handover from MBWA to WLAN. The number of trials is 4 . The measured values are ranged from $155 \mathrm{~ms}$ to $166 \mathrm{~ms}$. Table 2 shows the measured outage times in the handover from WLAN to MBWA. The measured values are ranged from $44 \mathrm{~ms}$ to 112 ms. Tables 1 and 2 show that the handover outage times from MBWA to WLAN and from WLAN to MBWA are around 160 


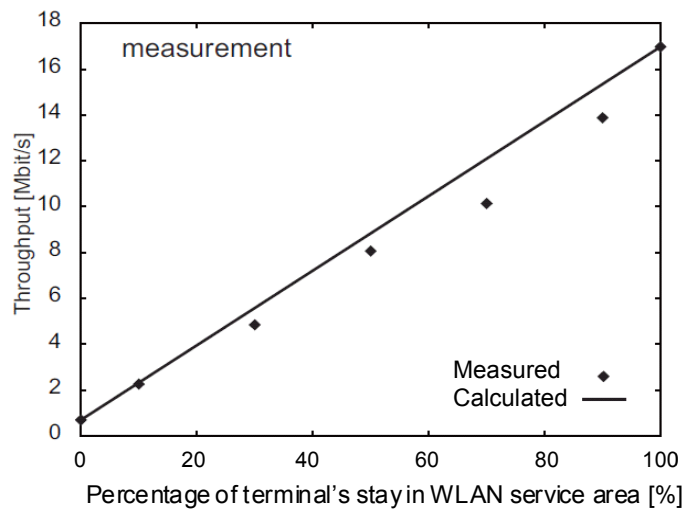

Figure 11 Measured throughput performance.

$\mathrm{ms}$ and $60 \mathrm{~ms}$, respectively. Since the same wired network accommodates the MBWA base-station and the WLAN access point, we consider that the outage time difference is caused by the performance difference between MBWA and WLAN such as a round trip time. The above results suggest that the proposed heterogeneous wireless network is feasible since the handover outage time is only less than $170 \mathrm{~ms}$, which is tolerable in a commercial service.

\section{Measured throughput performance}

In the field trial of the proposed heterogeneous wireless network, the mobile terminal preferentially selects the WLAN air interface with high throughput. Thus, it is expected that a higher percentage of terminal's stay in the WLAN service area yields a higher throughput. To confirm this, throughput measurements was carried out. Figure 11 shows the measured throughput performance. The vertical axis shows the measured throughput, and the horizontal axis shows the percentage of terminal's stay in the WLAN service area. $0 \%, 50 \%$, and $100 \%$ correspond 0 second, 5 seconds, and 10 seconds WLAN area stays in 10 seconds, respectively. The black diamond shows the measured performance. The solid line shows the calculated throughput performance, which is linearly interpolated by the uplink throughput of $690 \mathrm{kbit} / \mathrm{s}$ in MBWA for $0 \%$ and the uplink throughput of $16.9 \mathrm{Mbit} / \mathrm{s}$ in WLAN for $100 \%$, assuming the ideal handover without outage time. The values of $690 \mathrm{kbit} / \mathrm{s}$ and $16.9 \mathrm{Mbit} / \mathrm{s}$ are the maximum throughputs of MBWA and WLAN measured in the field trial. This figure shows that the measured throughput performance is almost the same as the calculated performance under the ideal handover condition.

\section{CONCLUSION}

This paper proposed a heterogeneous wireless communication network where both high throughput and wide coverage area are attained. No upgrades of wireless infrastructure and mobile terminals are required to construct the proposed network using the present homogeneous wireless networks since the handover functions are implemented as application programs on the content server and mobile terminals. We have carried out a field trial to confirm the feasibility of the proposed network. The field trial results show that the measured throughput performance is almost the same as the calculated performance assuming the ideal handover without outage time. We consider that the proposed network is suitable for smart phones with two air interfaces, where application programs can be installed. We also consider that the proposed network can provide a wireless data communication service with high throughput such as $1 \mathrm{Gbit} / \mathrm{s}$ and wide coverage area such as a cell radius of 1 or $2 \mathrm{~km}$ to users if LTE and 802.11ac air interfaces are applied to the proposed network though the highest throughput is achieved under a low mobility condition.

\section{ACKNOWLEDGEMENT}

The authors would like to thank Core Research for Evolutional Science and Technology (CREST) of the Japanese Science and Technology Agency (JST) for fund support.

\section{REFERENCE}

[1] M. Stemm and R. H. Katz, "Vertical Handoffs in Wireless Overlay Networks," ACM Mobile Networking (MONET), Special Issue on Mobile Networking in the Internet, vol.3, 1998, pp. 335-350.

[2] H. Schulzrinne and E. Wedlund, "Application-Layer Mobility Using SIP," ACM SIGMOBILE Mobile Computing and Communications Review, Volume 4, Issue 3, pp.47-57, 2000

[3] P. A. Pangalos, K. Boukis, L. Burness, A. Brookland, C. Beauchamps, A H. Aghvami, "End-to-end SIP based Real Time Application Adaptation During Unplanned Vertical Handovers," IEEE Global Communications Conference GLOBECOM '01, 2001.

[4] L. Ma, F. Yu, V. C. M. Leung, and T. Randhawa, "A New Method to Support UMTS/WLAN Vertical Handover Using SCTP," IEEE Wireless communications, August 2004, pp. 44-51.

[5] IEEE 802.21 Working Group, "IEEE 802.21 Overview of Standard for Media Independent Handover Services," 2006.

[6] S. Salsano, L. Veltri, G. Martiniello, and A. Polidoro, "Seamless Vertical Handover of VoIP Calls Based on SIP Session Border Controllers," IEEE International Conference on Communications ICC'06, 2006.

[7] H. Rutagemwa, S. Pack, X. Shen, and J. W. Mark, "Cross-layer Design and Analysis of Wireless Profiled TCP for Vertical Handover," IEEE International Conference on Communications ICC'07, 2007.

[8] R. Laroia, S. Uppala, and J. Li, "Designing a Mobile Broadband Wireless Access Network," IEEE Signal Processing Magazine, vol.21, no.5, pp. 20-28, 2004.

[9] H. Oguma, S. Kameda, N. Izuka, Y. Asano, Y. Yamazaki, T. Takagi, and K. Tsubouchi, "Measured Downlink Throughput Performance of MBWA System in Urban Area," IEEE International Symposium on Wireless Communication Systems ISWCS'08, 2008.

[10] N. Izuka, Y. Asano, Y. Yamazaki, H. Oguma, S. Kameda, T. Takagi, and K. Tsubouchi, "First-Ever Report on MBWA System Field Trial: Interference Issue in Sectored Cell Layout," IEEE Vehicular Technology Conference VTC'08 Fall, 2008.

[11] S. Kameda, H. Oguma, N. Izuka, Y. Asano, and Y. Yamazaki, T. Takagi, K. Tsubouchi, "Feasibility Study of Downlink Transmission with 256 QAM Based on Results of MBWA System Field Trial," European Wireless EW'09, 2009.

[12] H. Oguma, S. Kameda, N. Izuka, Y. Asano, Y. Yamazaki, T. Takagi, and K. Tsubouchi, "Uplink Throughput Performance of FH-OFDMA Improved by 16 QAM: Effect Estimation and Validation in MBWA System Field Trial," IEEE Personal, Indoor and Mobile Radio Communications Symposium PIMRC'09, 2009.

[13] H. Oguma, S. Kameda, N. Izuka, Y. Asano, Y. Yamazaki, T. Takagi, and K. Tsubouchi, "Coverage Estimation of Uplink 16 QAM Signal up to $20 \mathrm{MHz}$ Bandwidth Based on Field Trial Results of FH-OFDMA System," IEEE Wireless Communications and Networking Conference WCNC'10, 2010. 